\title{
Modeled Channel Distributions Explain Extracellular Recordings From Cultured Neurons Sealed to Microelectrodes
}

\author{
Jan Reinoud Buitenweg*, Wim L. C. Rutten, and Enrico Marani
}

\begin{abstract}
Amplitudes and shapes of extracellular recordings from single neurons cultured on a substrate embedded microelectrode depend not only on the volume conducting properties of the neuron-electrode interface, but might also depend on the distribution of voltage-sensitive channels over the neuronal membrane. In this paper, finite-element modeling is used to quantify the effect of these channel distributions on the neuron-electrode contact. Slight accumulation or depletion of voltage-sensitive channels in the sealing membrane of the neuron results in various shapes and amplitudes of simulated extracellular recordings. However, estimation of channel-specific accumulation factors from extracellular recordings can be obstructed by co-occuring ion currents and defect sealing. Experimental data from cultured neuron-electrode interfaces suggest depletion of sodium channels and accumulation of potassium channels.
\end{abstract}

Index Terms-Action potentials, extracellular stimulation, finite-element modeling, multielectrode arrays, neuron-electrode contact, voltage-sensitive channel densities.

\section{INTRODUCTION}

$\mathbf{T}$ HE ideal neuro-electronic interface for neuroprosthetic and neuroscientific application would provide a large number of two-way electrical contacts with single neurons. One of the aims of the neurotechnology program in our group is the development of these contacts by culturing neurons on top of microelectrode arrays (MEAs).

Extracellular recordings from cultured neurons on multielectrodes show a diversity of shapes and a wide range of amplitudes [1]-[4]. Furthermore, complete coverage, or sealing, of the electrode by neuron appeared to be favorable for extracellular recording [2]. This particular geometry of the neuron-electrode interface is characterized by a thin sealing gap between the

\footnotetext{
Manuscript received November 1, 2001; revised June 20, 2002. This work was part of the Neuro-Electronic Systems toward Tissue Implantation by Neural Grafting (NESTING) Project and was supported in part by the BIOMED II EC Project under Shared-Cost Contract BMH4-2723 and and in part by the the University of Twente under the OSF-Pioneer Fund. Asterisk indicates corresponding author.

*J. R. Buitenweg is with the Institute for Biomedical Technology, Signals and Systems Group, Faculty of Electrical Engineering, University of Twente, P.O. Box 217, 7500 AE Enschede, The Netherlands (e-mail: J.R.Buitenweg@el.utwente.nl)

W. L. C. Rutten is with the Institute for Biomedical Technology, Signals and Systems Group, Faculty of Electrical Engineering, University of Twente, Enschede, The Netherlands.

E. Marani is with the Institute for Biomedical Technology, Signals and Systems Group, Faculty of Electrical Engineering, University of Twente 7500 AE Enschede, The Netherlands. He is also with the Neuroregulation Group, Department of Neurosurgery, Leiden University Medical Center, Leiden, The Netherlands.

Digital Object Identifier 10.1109/TBME.2002.805555
}

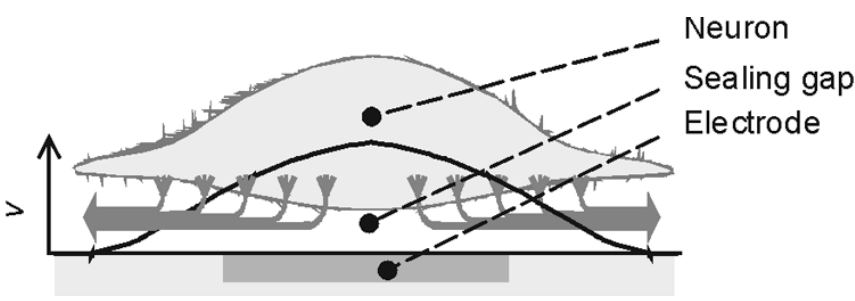

Fig. 1. Mediation of the neuron-electrode interface in extracellular recording. Due to current densities, arising from the neuronal membrane a potential distribution exists in the sealing gap which can be probed by the electrode.

neuronal membrane and the substrate. Bioelectrical activity can be observed due to a related potential distribution in this resistive gap, which is probed by the electrode (Fig. 1).

However, such a potential distribution only occurs if the bioelectrical activity results in a membrane current density into the sealing gap. It is demonstrated that this current density can be suppressed due to mutual canceling of ionic and capacitive membrane current densities. Total cancellation occurs if the voltage-sensitive ion channels are uniformly distributed over the membrane of the neuronal soma (which has no axon or dendrites) [5]. Hence, besides the mediating properties of the sealing gap also the distribution of ion channels might play a major role in extracellular recording.

In literature, several mechanisms are identified which affect the distribution of channels over the neuronal membrane. In the first place, the expression of channels is a dynamic process, as is the insertion and internalization of channels in the membrane [6]. Disturbance of this process, e.g., by enzymatical removal or axotomy due to dissociation during culturing, can affect the local distribution of channels [7]. Additionally, the inserted channels might migrate over the membrane due to diffusion or electrophoresis, or be immobilized to the cytoskeleton or to adhesion molecules involved in cell-cell couplings [8]-[11]. In both cases, the channel densities in the substrate adhesion region of a cultured neuron are likely to differ from the rest of the membrane, which will contribute to a potential distribution in the sealing gap.

For further development of neuron-electrode contacts, it is important to consider (1) if differences in channel distributions can explain the different shapes and amplitudes of extracellular recordings and (2) if the accumulation or depletion of channels in the adhesion region can be estimated from extracellular recordings. Therefore, finite-element modeling of the neuronelectrode interface is used to gain a quantitative insight into the influence of the distribution of voltage-sensitive channels 


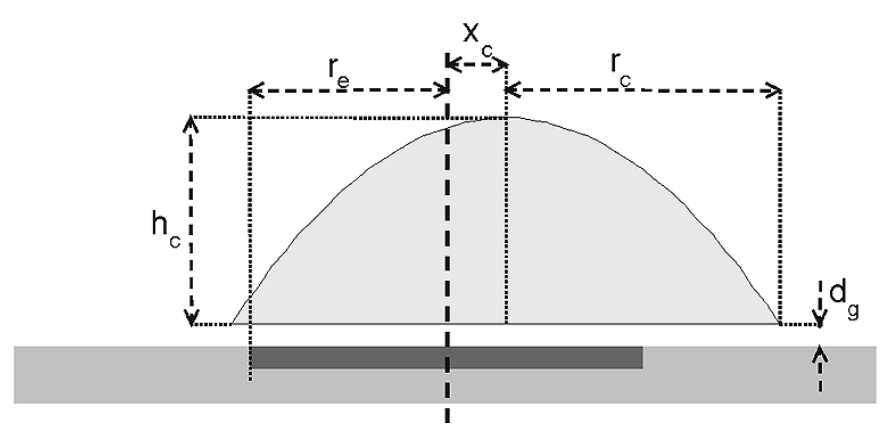

Fig. 2. Parametrical geometry of the neuron-electrode interface.

on extracellular recording. The model permits simulation of the neuron-electrode contact for a variety of interface geometries and includes the electrical behavior voltage-sensitive channels in the membrane. Accumulation or depletion of channels in the adhesion region is defined using a set of channel-specific accumulation factors, which are varied in several simulations. The possibility of estimating channel distributions from their contributions to the extracellular and intracellular recordings is considered. Finally, some experimentally obtained intracellular and extracellular recordings are presented and interpreted as an illustration.

\section{METHODS}

\section{A. Finite Element Model (FEM)}

In previous work, an FEM of the neuron-electrode interface is already presented in detail, assuming only passive membrane properties [12]. The mathematics of this model will be repeated briefly and extended by addition of voltage-sensitive channels in the membrane.

The neuron is modeled as a circular soma of radius $r_{c}$, with a parabolic height $\left(h_{c}\right)$ profile (Fig. 2). The neuron is positioned with an eccentricity $x_{c}$ on top of the electrode with radius $r_{e}$. A sealing gap of thickness $d_{g}$ is modeled between the soma and the substrate or the electrode. The axon and dendrites of the neuron are not included in the model.

The medium surrounding the interface, including the sealing gap, is represented by a three-dimensional volume conductor. This volume is meshed with tetrahedral shaped volume elements which permit numerical solutions of the Poisson equation

$$
\vec{\nabla} \cdot(\sigma \vec{\nabla} V)=0
$$

with $V$ the electrical potential and $\sigma=1.65 \mathrm{~S} / \mathrm{m}$ the conductivity of the medium. The nodes at the outer boundary of the meshed volume conductor are set to zero potential, representing a distant counter electrode.

The potentials at the membrane nodes are defined as transmembrane potentials, i.e., with respect to an intracellular potential $v_{i}$, and represented by a vector $\vec{v}_{m}=\left[v_{m, 1}, \ldots, v_{m, N_{m}}\right]^{T}$ with $N_{m}$ the number of membrane nodes. Similarly, the nodes at the electrode surface are defined as trans-electrode-electrolyte potentials, i.e., with respect to the electrode metal potential $v_{e}$, and represented by a vector $\vec{v}_{s}=\left[v_{s, 1}, \ldots, v_{s, N_{s}}\right]^{T}$, with $N_{s}$ the number of nodes at the electrode surface. The local currents into the membrane and electrode nodes, represented by the vectors $\vec{i}_{m}$ and $\vec{i}_{s}$ respectively, can be expressed as the sum of contributions from the local potentials, $\vec{v}_{m}$ and $\vec{v}_{s}$, and the stimulation currents injected to the electrode node or the intracellular node, $i_{e}$ and $i_{i}$

$$
\left[\begin{array}{c}
\vec{i}_{m} \\
\vec{i}_{s}
\end{array}\right]=K_{1} \cdot\left[\begin{array}{l}
\vec{v}_{m} \\
\vec{v}_{s}
\end{array}\right]+K_{2} \cdot\left[\begin{array}{l}
i_{e} \\
i_{i}
\end{array}\right]
$$

with $K_{1}$ and $K_{2}$ derived from the meshed volume conductor. In this paper, intracellular stimulation is applied by injecting a current, $i_{i}$, into the intracellular node. No current is injected into the node representing the electrode metal, so $i_{e}=0$. Furthermore, the electrode potential and intracellular potential are computed as

$$
\left[\begin{array}{l}
v_{i} \\
v_{e}
\end{array}\right]=K_{3} \cdot\left[\begin{array}{c}
\vec{v}_{m} \\
\vec{v}_{s}
\end{array}\right]+K_{4} \cdot\left[\begin{array}{c}
i_{e} \\
i_{i}
\end{array}\right]
$$

with $K_{3}$ and $K_{4}$ also derived from the meshed volume conductor.

Although it is known that the neuronal membrane can contain a large variety of different voltage-sensitive ion channels [14]-[17], a relatively simple description of a chick dorsal root ganglion (DRG) neuron is used, as presented by Bove et al. [3]. Only one type of sodium channel and one type of potassium channel is considered. Therefore, for each node $n=1, \ldots, N_{m}$, the time derivative of the local membrane potential is computed from the local membrane current and the ion-specific membrane current

$$
\begin{aligned}
\frac{d v_{m, n}}{d t}=\frac{1}{A_{m, n} \cdot c_{m}}\left[i_{m, n}-g_{\mathrm{Na}, n}\left(v_{m, n}-V_{\mathrm{Na}}\right)\right. \\
\left.\quad-g_{\mathrm{K}, n}\left(v_{m, n}-V_{\mathrm{K}}\right)-g_{\mathrm{Cl}, n}\left(v_{m, n}-V_{\mathrm{Cl}}\right)\right]
\end{aligned}
$$

with $A_{m, n}$ the membrane area represented by node $n$ and $c_{m}=$ $1 \mu \mathrm{F} / \mathrm{cm}^{2}$, the membrane capacity per unit area. In (4), the reversal potentials are set to $V_{\mathrm{Na}}=50 \mathrm{mV}, V_{\mathrm{K}}=-82 \mathrm{mV}$, and $V_{\mathrm{Cl}}=-65 \mathrm{mV}$ (according to [3]). The leakage conductance is computed as $g_{\mathrm{Cl}, n} \cdot \hat{g}_{\mathrm{Cl}}$, with $\hat{g}_{\mathrm{Cl}}=0.26 \mathrm{mS} / \mathrm{cm}^{2}$ (according to [3]). The local ion-specific voltage-sensitive sodium and potassium conductances are determined by the activation and inactivation factors $\left(m_{n}, h_{n}\right.$, and $\left.n_{n}\right)$ and by the local maximum ion-specific conductivities $\left(\hat{g}_{\mathrm{Na}}\right.$ and $\left.\hat{g}_{\mathrm{K}}\right)$

$$
\begin{aligned}
g_{\mathrm{Na}, n} & =A_{m, n} \cdot m_{n}^{3} \cdot h_{n} \cdot \hat{g}_{\mathrm{Na}} \\
g_{\mathrm{K}, n} & =A_{m, n} \cdot n_{n}^{4} \cdot \hat{g}_{\mathrm{K}} .
\end{aligned}
$$

Nonuniform channel distributions will result in altered local maximum $\mathrm{Na}^{+}$and $\mathrm{K}^{+}$conductivities, $\hat{g}_{\mathrm{Na}}$ and $\hat{g}_{\mathrm{K}}$. In this paper, the accumulation or depletion of channels in the adhesion region of the membrane are defined in terms of the average membrane properties as are observed using whole cell recording techniques

$$
\begin{aligned}
\hat{g}_{\mathrm{Na}} & =\gamma_{\mathrm{Na}} \cdot \tilde{g}_{\mathrm{Na}} \\
\hat{g}_{\mathrm{K}} & =\gamma_{\mathrm{K}} \cdot \tilde{g}_{\mathrm{K}}
\end{aligned}
$$

with channel-specific accumulation factors $\gamma_{\mathrm{Na}}$ and $\gamma_{\mathrm{K}}$ and average ion-specific conductivities $\tilde{g}_{\mathrm{Na}}$ and $\tilde{g}_{\mathrm{K}}$. As a consequence, 
the maximum conductivities in the upper membrane are determined by

$$
\begin{gathered}
\hat{g}_{\mathrm{Na}}=\frac{A_{m}-\gamma_{\mathrm{Na}} \cdot A_{j m}}{A_{f m}} \cdot \tilde{g}_{\mathrm{Na}} \\
\hat{g}_{\mathrm{K}}=\frac{A_{m}-\gamma_{\mathrm{K}} \cdot A_{j m}}{A_{f m}} \cdot \tilde{g}_{\mathrm{K}} .
\end{gathered}
$$

The definitions in (6) and (7) result in the average ion-specific conductivities, $\tilde{g}_{\mathrm{Na}}$ and $\tilde{g}_{\mathrm{K}}$, as long as

$$
A_{m}=\sum_{n=1}^{N_{m}} A_{m, n}=A_{f m}+A_{j m}, \quad 0 \leq \gamma \leq \frac{A_{m}}{A_{j m}}
$$

and $A_{m}$ the total membrane area, $A_{f m}$ the area of the free part of the membrane, and $A_{j m}$ the area of the adhesion region. The average ion-specific conductivities $\tilde{g}_{\mathrm{Na}}=15 \mathrm{mS} / \mathrm{cm}^{2}, \tilde{g}_{\mathrm{K}}=$ $3.2 \mathrm{mS} / \mathrm{cm}^{2}$ are used, according to [3].

The time derivatives of the local $\mathrm{Na}^{+}$inactivation and $\mathrm{K}^{+}$ activation constants, $h_{n}$ and $n_{n}$, respectively, are computed as

$$
\begin{aligned}
\frac{d h_{n}}{d t} & =\frac{\lambda_{h}\left(h_{\infty}\left(v_{m, n}\right)-h_{n}\right)}{\tau_{h}\left(v_{m, n}\right)} \\
\frac{d n_{n}}{d t} & =\frac{\lambda_{n}\left(n_{\infty}\left(v_{m, n}\right)-n_{n}\right)}{\tau_{n}\left(v_{m, n}\right)}
\end{aligned}
$$

with the description of the channel dynamics by $\lambda_{h}, \lambda_{n}, \lambda_{\infty}(v), n_{\infty}(v), \tau_{h}(v)$ and $\tau_{n}(v)$ adopted from the above-mentioned chick DRG model [3]. The local $\mathrm{Na}^{+}$ activation constant, $m_{n}$, is considered to be very fast and is, therefore, computed directly from $v_{m, n}$ as $m_{n}=m_{\infty}\left(V_{m, n}\right)$ according to the same model.

The time derivatives of the local potentials at the electrode surface are computed for each node, $n=1, \ldots, N_{s}$ assuming a capacitive nature of the platinized electrode

$$
\frac{d v_{s, n}}{d t}=\frac{i_{s, n}}{A_{s, n} \cdot c_{s}}
$$

with $a_{s, n}$ the electrode area represented by node $n$ and $c_{s}=$ $500 \mu \mathrm{F} / \mathrm{cm}^{2}$ the electrode capacity per unit area, based on an impedance of $400 \mathrm{k} \Omega$ at $1 \mathrm{kHz}$, of a platinized electrode of $10-\mu \mathrm{m}$ diameter.

The total set of partial difference equations (PDEs) is solved numerically in MATLAB version 5.3 (The Mathworks, Inc., Natick, MA), using a variable order solver, based on numerical differentiation formulas, (ODE15s) with a maximum time-step of $10 \mu \mathrm{s}$. The initial conditions at $t=0$ were taken $\vec{v}_{m}=-$ $65 \mathrm{mV}$ and $\vec{v}_{s}=0$. After solving the set of PDEs, all ionspecific and capactitive membrane currents can be extracted from the computed membrane potentials and channel activation constants.

\section{B. Estimation of Channel Distributions}

For evaluation and improvement of neuron-electrode contacts it is important to estimate the accumulation or depletion of channels in the adhesion region. In previous work, the FEM model is used for studying the effect of geometry on intracellular and extracellular stimulation and recording [12]. It was demonstrated that, in absence of voltage-sensitive channels, the extracellular response, $V_{e}^{\text {(passive) }}$, to an intracellular stimulus current, $I_{\text {stim }}$, can be described using the sealing resistance, $R_{\text {seal }}$, and the electrical equivalent fraction of the membrane, $\alpha$, which is probed by the electrode

$$
V_{e}^{(\text {passive })}=\alpha \cdot R_{\text {seal }} \cdot I_{\text {stim }}=\alpha \cdot R_{\text {seal }} \cdot A_{m} \cdot i_{m}^{\text {(passive })}
$$

with $i_{m}^{\text {(passive) }}$ the (assumed to be uniformly distributed) membrane current density without open channels, due to the stimulation current. It is clear that the exact current density distribution can not be reconstructed from one extracellular potential, $V_{e}$. Instead, (11) is used for determination of the effective uniformly distributed current density through the lower membrane, required to explain the total measured extracellular response (i.e., including the contributions from ion channels)

$$
\vec{i}_{j m}=\frac{V_{e}}{\alpha \cdot R_{\text {seal }} \cdot A_{m}} .
$$

The neuron-electrode contact is simulated for several $\mathrm{Na}^{+}$ channel densities in the lower membrane: uniform distribution $\left(\gamma_{\mathrm{Na}}=1\right)$, depletion $\left(\gamma_{\mathrm{Na}}=0.8\right)$ and accumulation $\left(\gamma_{\mathrm{Na}}=1.2\right)$. The results are organized in columns for each case (Fig. 3) and include $\mathrm{Na}^{+}$and capacitive current density distributions, the total current density distribution, the distribution of extracellular potentials around the neuron and the intracellular and extracellular potentials. In all cases, an action potential is evoked by intracellular stimulation with a rectangular current pulse of $100 \mathrm{pA}$. The shape of this intracellular action potential is not affected by the local changes in the $\mathrm{Na}^{+}$channel density since the total number of channels in the membrane is not altered, due to the definitions in (7) and (8). The ionic contribution to this current density can be obtained by subtraction of a capacitive component, which is also assumed to be uniformly distributed and can be determined using the derivative of the intracellular potential and the membrane capacity per unit area

$$
\vec{i}_{j m, \text { ion }}=\vec{i}_{j m}-i_{c m}=\vec{i}_{j m}-c_{m} \frac{\partial V_{\text {intra }}}{\partial t} .
$$

This effective ionic current density is ruled by the accumulation and/or depletion of the ion channels in the lower membrane and can also be expressed as the sum of all separate ion-specific current densities, scaled to the accumulation factors defined in (6)

$$
\vec{i}_{j m, \text { ion }}=\gamma_{\mathrm{Na}} \cdot \tilde{g}_{\mathrm{Na}}\left(V_{\text {intra }}-V_{\mathrm{Na}}\right)+\gamma_{\mathrm{K}} \cdot \tilde{g}_{\mathrm{K}}\left(V_{\text {intra }}-V_{\mathrm{K}}\right)
$$

Accumulation or depletion of channels in the sealing gap will result in a deviation of the effective lower membrane ionic current density from the average ionic current density, which is the sum of all average ion-specific current densities

$$
\tilde{i}_{m, \text { ion }}=\tilde{g}_{\mathrm{Na}}\left(V_{\text {intra }}-V_{\mathrm{Na}}\right)+\tilde{g}_{\mathrm{K}}\left(V_{\text {intra }}-V_{\mathrm{K}}\right)
$$

this average ionic current density can be estimated from the stimulus current and the derivative of the intracellular potential, according to

$$
\tilde{i}_{m, \text { ion }}=\tilde{i}_{m}-i_{c m}=\frac{I_{\text {stim }}}{A_{m}} c_{m} \frac{\partial V_{\text {intra }}}{\partial t} .
$$

As a consequence of (14) and (15), the ratio between the current densities, as determined using (13) and (16), contains information about the effective accumulation or depletion of ion chan- 


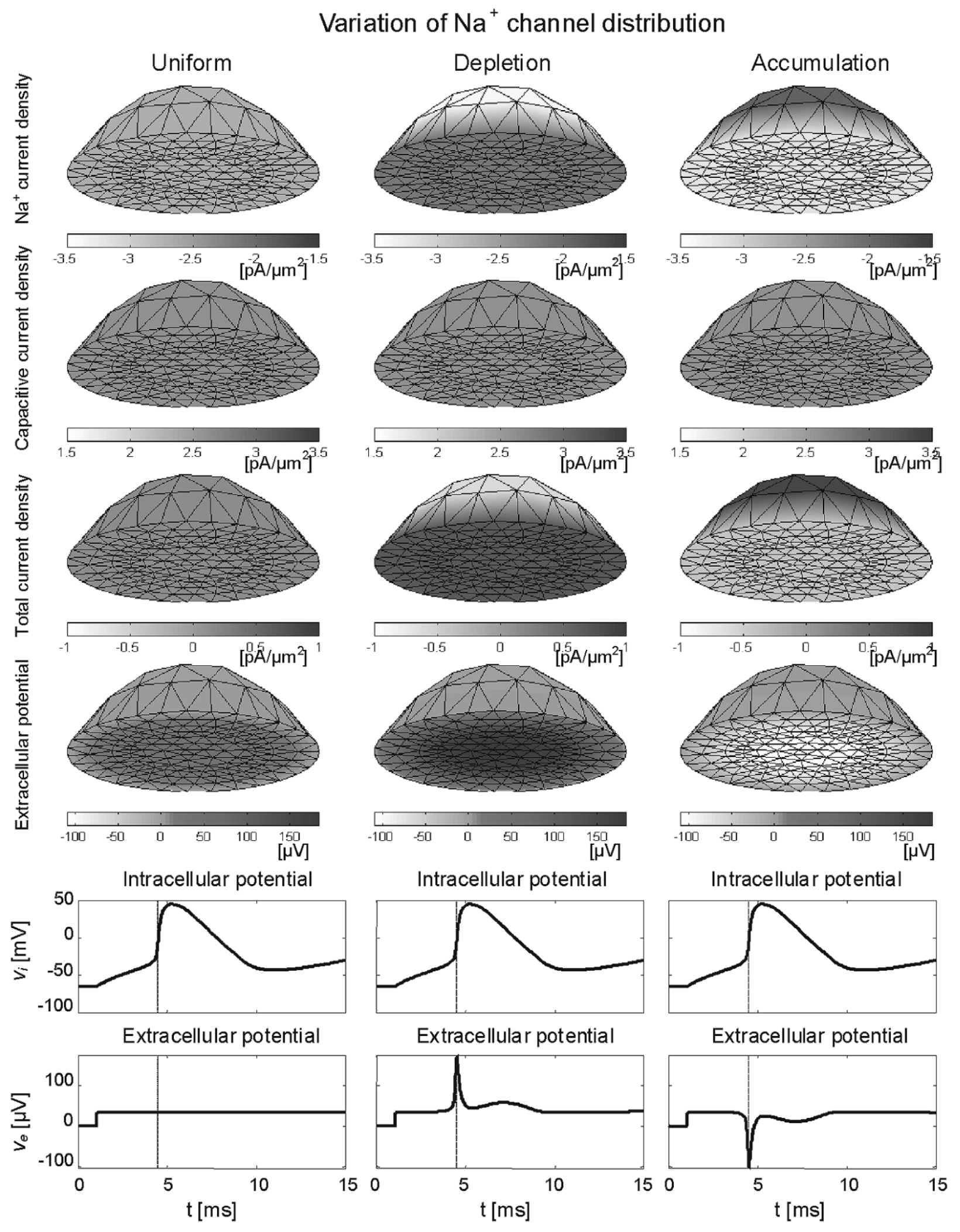

Fig. 3. Simulation of the neuron-electrode contact for three distributions of voltage-sensitive $\mathrm{Na}^{+}$channels. Geometry parameters: $r_{c}=10 \mu \mathrm{m}, h_{c}=7 \mu \mathrm{m}$, $r_{e}=5 \mu \mathrm{m}, x_{c}=0$, and $d_{g}=50 \mathrm{~nm}$. (see Fig. 2). First column: Uniform channel distribution $\left(\gamma_{\mathrm{Na}}=1\right)$. Second column: Depletion of Na ${ }^{+}$channels $\left(\gamma_{\mathrm{Na}}=0.8\right)$. Third column: Accumulation of $\mathrm{Na}^{+}$channels $\left(\gamma_{\mathrm{Na}}=1.2\right)$. All other membrane parameters are uniformly distributed over the total membrane area. Intracellular stimulation is applied, using a rectangular current pulse of $100 \mathrm{pA}$ starting at $t=1 \mathrm{~ms}$. In the upper three rows, local current densities at $t=4.5 \mathrm{~ms}$ (marked by the vertical lines in the diagrams) are represented by the colored regions on the cellular surfaces (from top to bottom: sodium, capacitive, and total current density, respectively). In the fourth row, the extracellular potential distribution at $t=4.5 \mathrm{~ms}$ is depicted. Below each column, the intracellular and extracellular potentials are plotted versus time. 
nels in the lower membrane. Therefore, an accumulation factor, $\gamma$, is estimated as

$$
\gamma=\frac{\tilde{i}_{j m, \text { ion }}}{\tilde{i}_{m}}
$$

If only $\mathrm{Na}^{+}$channels are open, i.e., $\tilde{g}_{\mathrm{Na}} \gg \tilde{g}_{\mathrm{K}}$, then $\mathrm{Na}^{+}$currents dominate in (14) and (15) and the estimated accumulation factor, $\gamma$, reflects the $\mathrm{Na}^{+}$accumulation factor, $\gamma_{\mathrm{Na}}$. Similarly, if only $\mathrm{K}^{+}$channels are open, i.e., $\tilde{g}_{\mathrm{K}} \gg \tilde{g}_{\mathrm{Na}}$, the $\mathrm{K}^{+}$accumulation factor, $\gamma_{\mathrm{K}}$, is reflected. It should be noted that if more channel types are open at the same time, the estimated accumulation factor must be regarded as a weighted average of multiple channel accumulation factors.

\section{Experiments}

Dorsal root ganglions are dissected from neonatal (P3) rats, enzymatically dissociated (collagenase and trypsin) and plated on a MEA with 61 hexagonally ordered electrodes of $10 \mu \mathrm{m}$ in diameter (impedance $200-500 \mathrm{k} \Omega$ at $1 \mathrm{kHz}$ ). From observation using phase contrast microscopy, electrodes are selected which are completely covered by a neuron. Sealing resistances are measured using impedance spectroscopy. The intracellular potential of the neuron is measured at room temperature $\left(23^{\circ} \mathrm{C}\right)$ using a whole cell current clamp configuration [13]. Pipette filling solution contains (in $\mathrm{mM}$ ): $140 \mathrm{KCl}, 10 \mathrm{NaCl}, 1 \mathrm{CaCl}_{2}$, $2 \mathrm{MgCl}_{2}, 10$ EGTA, 10 HEPES 2 ATP-Mg, pH 7.2. The extracellular solution contains (in $\mathrm{mM}$ ): $140 \mathrm{NaCl}, 5 \mathrm{KCl}, 1 \mathrm{CaCl}_{2}$, 6 Glucose, $1 \mathrm{MgCl}_{2}, 10 \mathrm{HEPES}, \mathrm{pH}$ 7.2. Intracellular stimulation is applied by injection of rectangular current pulses of $150 \mathrm{~ms}$, ranging from 300 to $760 \mathrm{pA}$. The intracellular and extracellular responses are digitized and stored for offline analysis.

\section{RESULTS}

\section{A. Influence of Channel Distributions}

During the rising phase of the action potential, inward $\mathrm{Na}^{+}$ current densities occur over the entire membrane, depending on the $\mathrm{Na}^{+}$channel distribution (top row). The capacitive current density, driven by the derivative of the membrane potentials opposes the $\mathrm{Na}^{+}$currents (second row). At this moment, the contribution of other ionic current densities $\left(\mathrm{K}^{+}\right.$and $\left.\mathrm{Cl}^{-}\right)$to the total current density is very small, so the resulting membrane current density is mainly determined by the sum of the $\mathrm{Na}^{+}$ and capacitive current densities (third row). The total current through the membrane, which is the sum of all ionic and capacitive currents is ruled by the injected stimulation current.

When the $\mathrm{Na}^{+}$channels are uniformly distributed $\left(\gamma_{\mathrm{Na}}=\right.$ 1 , first column), the local $\mathrm{Na}^{+}$and capacitive current densities will compensate each other, resulting in a uniformly distributed membrane current density without relation to the bioelectrical activity of the neuron. Due to this current density, a potential distribution builds up in the sealing gap, which depends only on the stimulus current. As a result, no bioelectrical activity is observed in the extracellular recording.

When local differences in $\mathrm{Na}^{+}$channel densities are introduced, the magnitude of the $\mathrm{Na}^{+}$current density also differs locally over the membrane. However, the capacitive current den- sity is still (almost) uniformly distributed, since the differences in the (derivatives) of the local transmembrane potentials remain small. Hence, these two current densities will not compensate locally, resulting in a $\mathrm{Na}^{+}$-dependent current density in the sealing gap. With $\mathrm{Na}^{+}$depletion $\left(\gamma_{\mathrm{Na}}=0.8\right.$, second column), the magnitude of the capacitive current density locally exceeds the $\mathrm{Na}^{+}$current density and an outward current density occurs, resulting in a positive spike in the extracellular recording. Similarly, accumulation of $\mathrm{Na}^{+}\left(\gamma_{\mathrm{Na}}=1.2\right.$, third column) results in a negative spike.

Both shape and amplitude of the extracellular recording depend on the distribution of channels, as appears from simulations with several accumulation factors for $\mathrm{Na}^{+}$and $\mathrm{K}^{+}$channels (Fig. 4). Although the intracellular potential is not affected by the $\mathrm{Na}^{+}$and $\mathrm{K}^{+}$channel distributions (top row), the extracellular recordings differ (second row). A nonuniform $\mathrm{Na}^{+}$ channel distribution appears as a fast peak during the rising phase of the intracellular action potential followed by a slow wave during the falling phase. This positive slow wave is the result of inward $\mathrm{Na}^{+}$current densities due to the still activated $\mathrm{Na}^{+}$channels (full inactivation at $t=11 \mathrm{~ms}$ ) combined with an increasing difference between the membrane potential andthe $\mathrm{Na}^{+}$equilibrium potential $\left(V_{\mathrm{Na}}=50 \mathrm{mV}\right)$. Nonuniform $\mathrm{K}^{+}$ channel distributions result in a more slow wave during the falling phase of the action potential, which occur in these simulations at the same time as the slow $\mathrm{Na}^{+}$wave described above. Contrary to depletion of $\mathrm{Na}^{+}$channels, a depletion of $\mathrm{K}^{+}$channels in the lower membrane results in a negative wave in the extracellular response.

\section{B. Estimation of Channel Distributions}

For interpretation of experimental data it would be useful to know to what extend the distributions of different ion channels, i.e., accumulation or depletion in the sealing gap, can be estimated from intracellular and extracellular recordings. For this purpose, the average ionic current density and the effective lower membrane ionic current densities are computed using (12), (13) and (16). The required sealing resistance, $R_{\text {seal }}=3.12 \mathrm{M} \Omega$, and electrical equivalent fraction of the membrane, $\alpha=0.123$, was computed in previous work for the same geometry of the neuron-electrode interface [12]. The results are plotted in the third row of Fig. 4. During the rising phase of the action potential, fast negative current density peaks occur due to the influx of $\mathrm{Na}^{+}$ions. The slow positive waves during the falling phase of the action potential are mainly due to outward $\mathrm{K}^{+}$current densities. A depletion of $\mathrm{Na}^{+}$channels causes a deviation of the lower membrane ionic current densities $\left(\tilde{i}_{j m, \text { ion }}\right.$ at $\left.\gamma_{\mathrm{Na}}=0.2, \ldots, 0.8\right)$ from the average ionic current density $\left(\tilde{i}_{m \text {,ion }}\right)$ during almost the entire action potential [Fig. 4(a)]. Variation of the $\mathrm{K}^{+}$channel densities $\left(\tilde{i}_{j m \text {,ion }}\right.$ at $\left.\gamma_{K}=0.2, \ldots, 1.6\right)$ only affects the slow waves of the lower membrane ionic current density, the fast peak during the rising phase of the intracellular potential does not differ from the average ionic current density. This means that no $\mathrm{K}^{+}$currents occur during the fast peak and that this peak is only determinedby the $\mathrm{Na}^{+}$and the $\mathrm{Cl}^{-}$current density (of which the latter is negligibly small: $g_{\mathrm{Na}}=15 \mathrm{mS} / \mathrm{cm}^{2}$ and $g_{\mathrm{Cl}}=0.3 \mathrm{mS} / \mathrm{cm}^{2}$ ). 

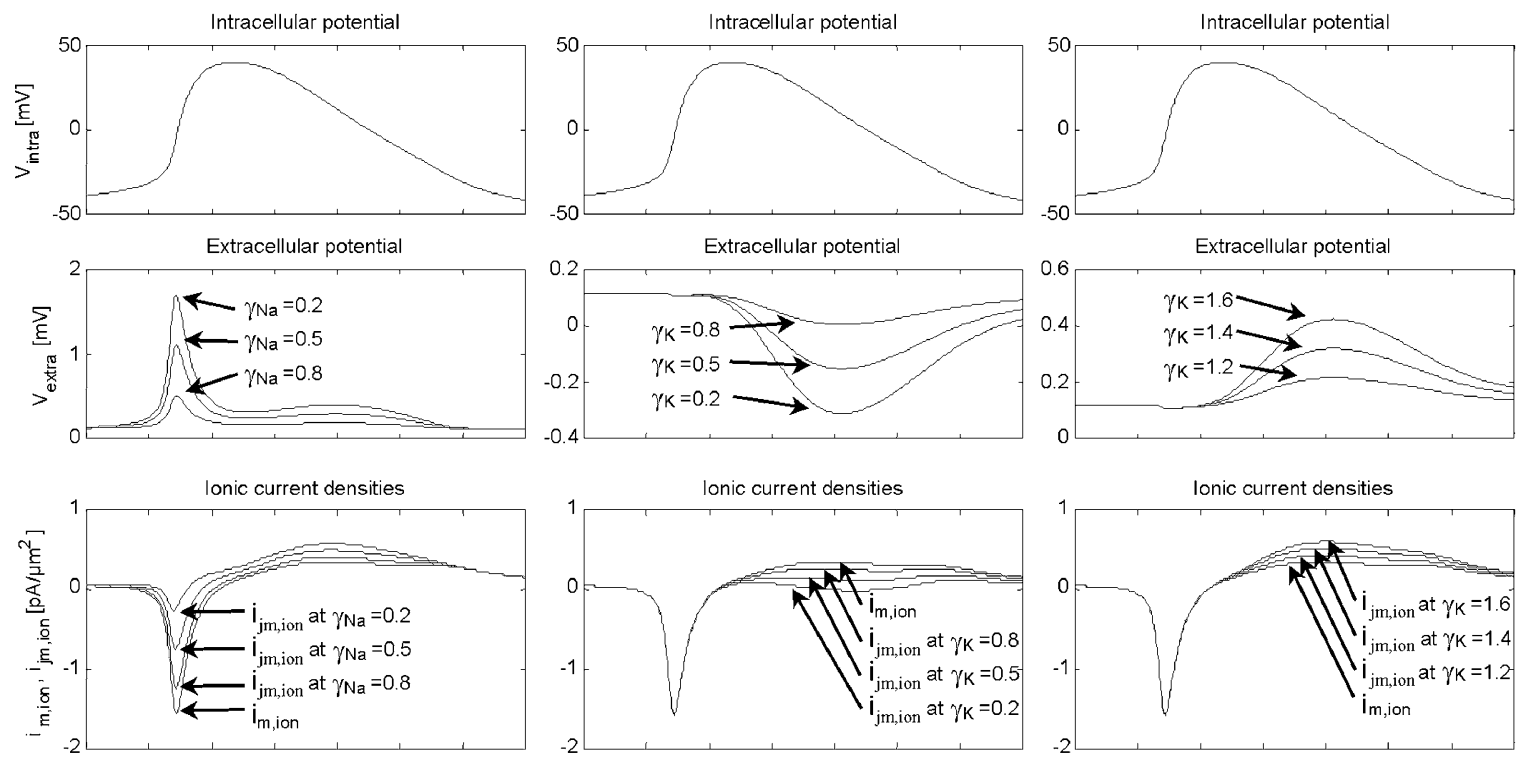

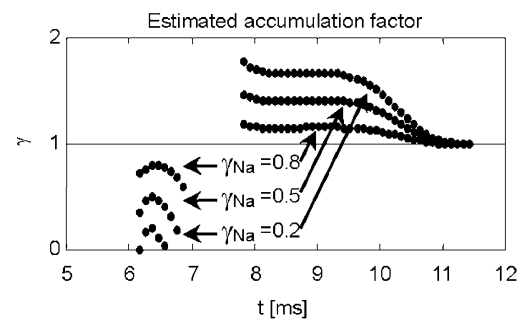

(a)

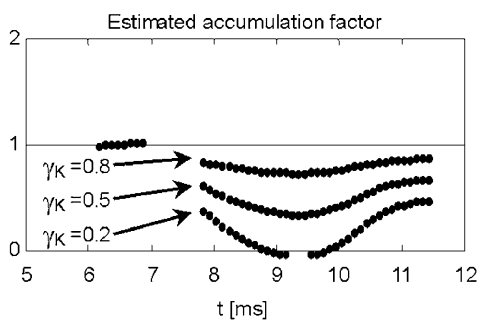

(b)

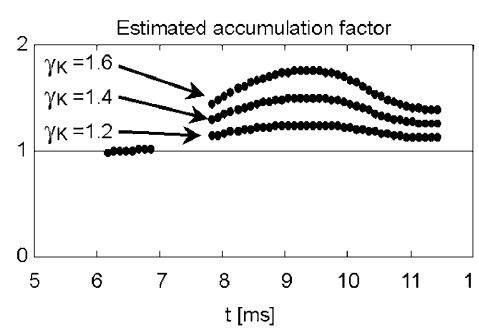

(c)

Fig. 4. Simulation of the intracellular and extracellular recordings with different distributions of $\mathrm{Na}^{+}$and $\mathrm{K}^{+}$. Geometry parameters: $r_{c}=20 \mu \mathrm{m}, h_{c}=14 \mu \mathrm{m}$, $r_{e}=5 \mu \mathrm{m}, x_{c}=0$ and $d_{g}=50 \mathrm{~nm}$ (see Fig. 2). (a) Depletion of $\mathrm{Na}^{+}$channels $\left(\gamma_{\mathrm{Na}}=0.2, \ldots, 0.8\right)$. (b) Depletion of $\mathrm{K}^{+}$channels (solid line: $\gamma_{\mathrm{K}}=$ $0.2, \ldots, 0.8$ ). (c) Accumulation of $\mathrm{K}^{+}$channels (solid line: $\gamma_{\mathrm{K}}=1.2, \ldots, 1.6$ ). The average ionic current density, $i_{m}$, ion, and the lower membrane current density, $i_{j m, \text { ion }}$, are computed from the intracellular and extracellular potentials using (12), (13), and (16). From these current densities, the estimated accumulation factor, $\gamma$, is computed using (17).

The derived ionic current densities $\tilde{i}_{m \text {,ion }}$ and $\tilde{i}_{j m \text {,ion }}$ are used for estimation of the accumulation factor, $\gamma$, according to (17) (Fig. 4, fourth row). To avoid numerical noise, $\gamma$ is only computed at times at which $\tilde{i}_{m \text {,ion }}>0.2 \mathrm{pA} / \mu \mathrm{m}^{2}$. The estimated accumulation factors appear to vary as a function of time. In Fig. 4(a), the estimated accumulation factor reaches the simulated accumulation factor $\gamma_{\mathrm{Na}}$ at the minimum of the fast peak. During the slow wave, however, a channel accumulation of $\gamma=$ 1.7 is computed, which deviates from the simulated accumulation factor. This erroneous value is due to the outward $\mathrm{K}^{+}$ membrane current densities which during the slow wave also contribute to $\tilde{i}_{m \text {,ion }}$ and $\tilde{i}_{j m \text {,ion. Therefore, the estimated accu- }}$ mulation factor should be interpreted with respect to the $\mathrm{Na}^{+}$ accumulation factor when no other ionic currents are present, i.e., at the minimum of the fast peak. This also holds in Fig. 4(b), (c) where $\gamma_{\mathrm{Na}}=1$. The co-occurence of several ionic current densities will also cause errors when $\gamma$ is used for the estimation of $\gamma_{K}$, as can be seen from Fig. 4(b), (c). Both the depletion and the accumulation of channels are biased by the inward $\mathrm{Na}^{+}$ current density (and probably the leakage current density) and $\gamma$ does not reflect $\gamma_{K}$ properly.

\section{Influence of Geometry}

From previous work it is known that, besides the distribution of channels, also the geometry of the neuron-electrode in- terface affects the extracellular recording. In order to see how the estimation of channel distributions is affected by the interface geometry, simulations are performed in which the geometry is varied from complete to defect sealing. In Fig. 5, a neuron with a radius of $r_{c}=20 \mu \mathrm{m}$ is shifted of an electrode of radius $r_{e}=5 \mu \mathrm{m}$. An accumulation factor of $\gamma_{\mathrm{Na}}=0.5$ is assumed to ensure a substantial extracellular recording.

The extracellular recording is simulated at several eccentricities from complete $\left(x_{c}=0\right.$ and $\left.x_{c}=12 \mu \mathrm{m}\right)$ to defect sealing ( $x_{c}=18 \mu \mathrm{m}$ and $\left.x_{c}=27 \mu \mathrm{m}\right)$. When the eccentricity increases, the amplitude of the extracellular recording decreases. At $x_{c}=18 \mu \mathrm{m}$, the neuron still covers the electrode partially, resulting in a small extracellular response. This response disappears when the coverage expires $\left(x_{c}=27 \mu \mathrm{m}\right)$.

Although the extracellular recordings decrease in amplitude, the computed average ionic current densities do not change as long as the sealing remains complete. However, a defect sealing causes an increase in the observed $\tilde{i}_{j m \text {,ion }}$, especially during the fast peak $\left(x_{c}=18 \mu \mathrm{m}\right)$. This would corrupt the estimated accumulation factor and especially its interpretation concerning $\gamma_{\mathrm{Na}}$. At $x_{c}=27 \mu \mathrm{m}, \tilde{i}_{m \text {,ion }}$ and $\tilde{i}_{j m \text {,ion }}$ are almost equal, suggesting an accumulation factor of $\gamma=1$ during the entire actionpotential. This does not surprise since the passive coupling $\left(\alpha \cdot R_{j}\right)$ is almost annihilated, which impedes the observability of the channel distributions. However, for small sealing defects, 

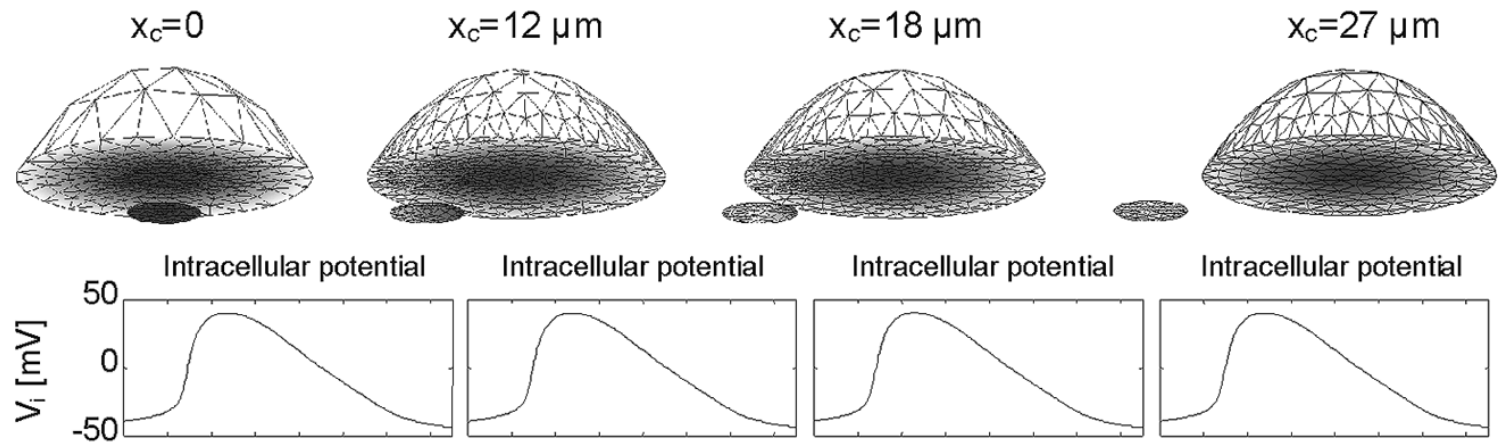

Intracellular potential

Intracellular potential
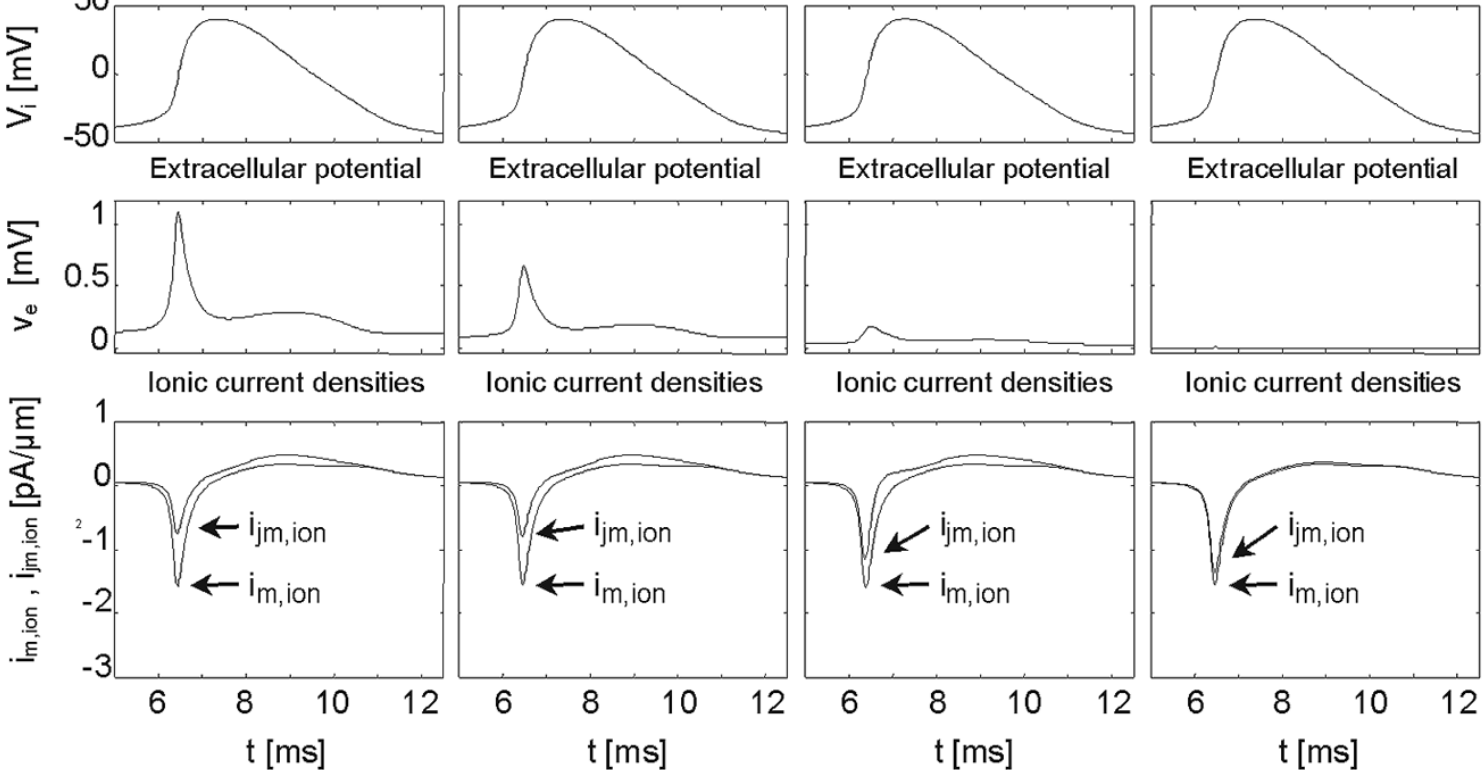

Extracellular potential
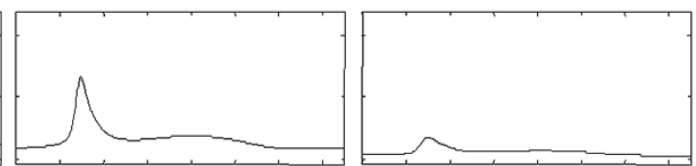

Ionic current densities

Ionic current densities

Ionic current densities
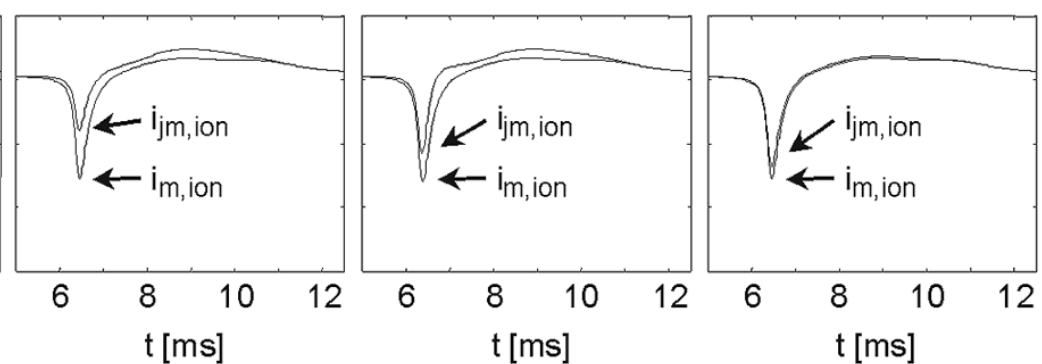

Fig. 5. Simulation of the neuron-electrode contact for geometries from complete ( $x_{c}=0$ and $\left.12 \mu \mathrm{m}\right)$ to defect sealing $\left(x_{c}=18\right.$ and $\left.27 \mu \mathrm{m}\right)$. Other geometry parameters are the same as in Fig. 4. Action potentials are elicited by intracellular stimulation with $300 \mathrm{pA}$ (first row). Due to depletion of $\mathrm{Na}^{+}$channels $\left(\gamma_{\mathrm{Na}}=\right.$ 0.5 ), bioelectrical activity is observed in the extracellular recordings (second row) which decreases with the eccentricity of the neuron. From the intracellular and extracellular potential, the average and lower membrane ionic current densities are estimated (third row).

as with $x_{c}=18$, this passive coupling is still present in certain extent $\left(\alpha \cdot R_{\text {seal }}=0.08 \mathrm{mV} / \mathrm{pA}\right.$, computed for this geometry in [12]) and should be sufficient for extracellular recording. The erroneous observation of $\tilde{i}_{j m \text {,ion }}$ is due to the interaction between the extracellular volume conductor (including the sealing gap) and the electrode-electrolyte interface. At $x_{c}=18 \mu \mathrm{m}$, part of the electrode surface is sealed by the neuron and the free part of the surface contacts the extracellular space. Due to the capacitive nature of the electrode-electrolyte interface, high-frequency currents from the lower membrane can escape from the sealing gap by entering the electrode through the sealed part and leaving through the free part of the surface. The contribution of these currents to the potential distribution in the sealing gap is, therefore, less than of low-frequency currents. This leakage effect depends on the electrode impedance, as can be seen from Fig. 6 . These simulation results are obtained with electrode impedance varying from $200 \mathrm{k} \Omega$ to $2 \mathrm{M} \Omega$ at $1 \mathrm{kHz}$ $\left(c_{e}=100, \ldots, 1000 \mu \mathrm{F} / \mathrm{cm}^{2}\right)$. The extracellular responses decrease with decreasing electrode impedance and also loose their high-frequency content. The slow wave, following the fast peak, is not affected.

\section{Experimental Data}

As an illustration of the relevance of the simulated results, some experimental data is added. The culturing procedure resulted in healthy neuronal cultures on MEAs. However, the probability of complete coverage was very low. In Fig. 7,
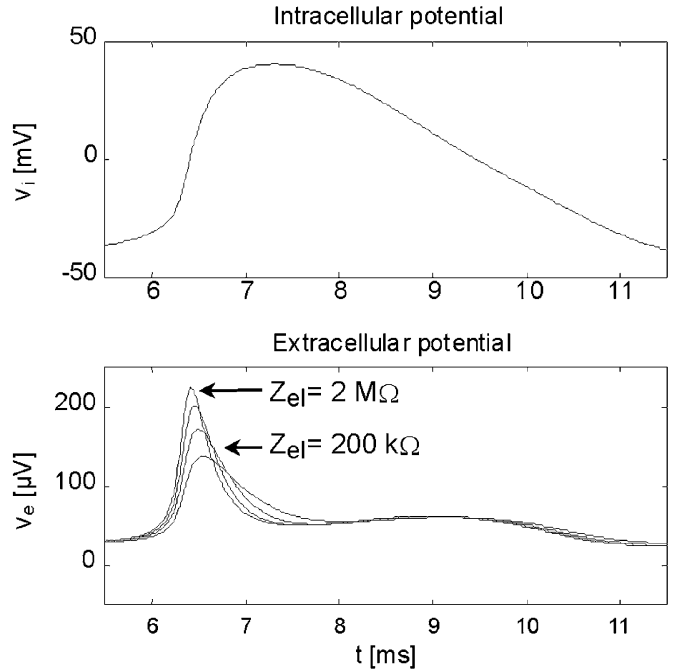

Fig. 6. Effect of electrode impedance on extracellular recording for the same geometry as used in Fig. 4 (defect sealing, $x_{c}=18 \mu \mathrm{m}$ ). While the intracellular recording remains unchanged, the fast peak in the extracellular recording (bottom trace) is affected when the electrode impedance is decreased from $2 \mathrm{M} \Omega$ to $200 \mathrm{k} \Omega$ at $1 \mathrm{kHz}$.

three series of current clamp measurements are presented together with the extracellular response. These measurements are obtained from two cultured neuron-electrode interfaces at 1 DIV. From neuron 1, the observed extracellular shapes changed during the patch clamp and are, therefore, presented 

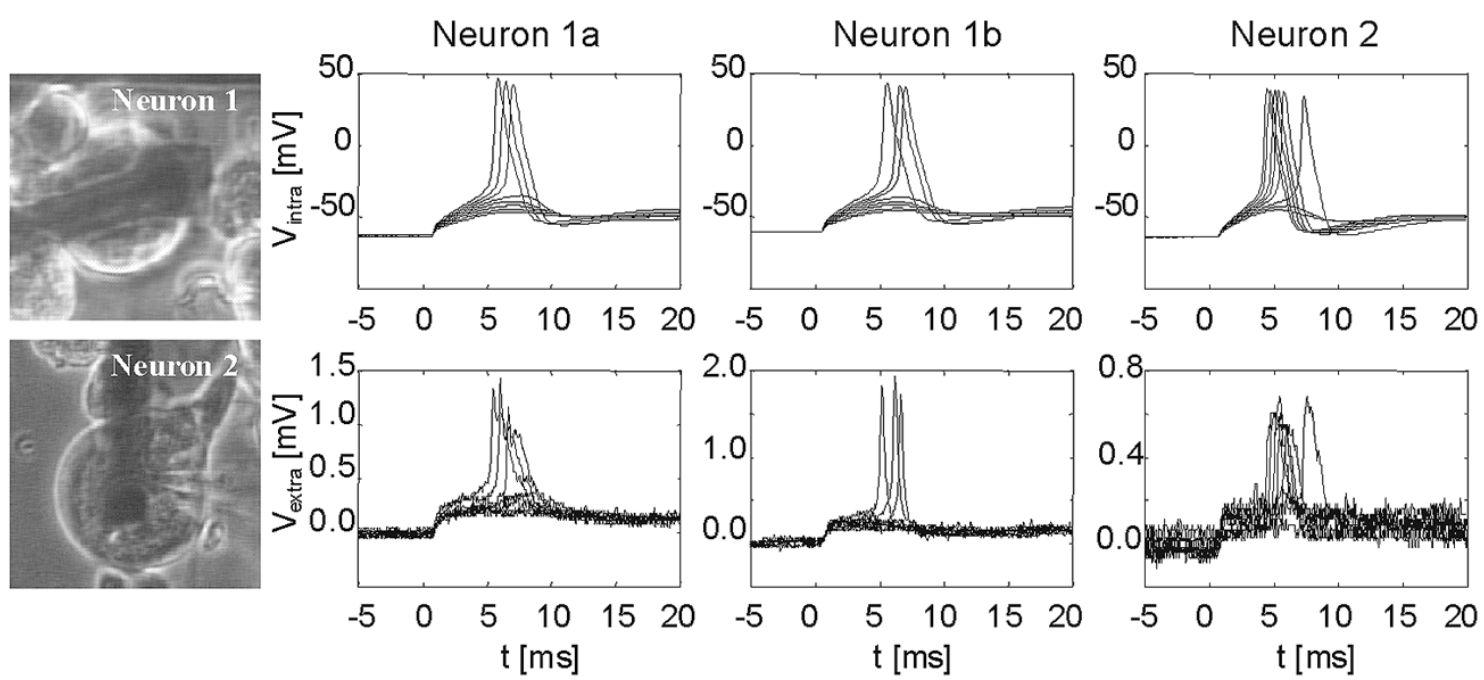

Fig. 7. Measured intracellular and extracellular responses from two rat DRG neurons, cultured on a microelectrode (microphotographs). The extracellular recordings of neuron 1 changed during patch clamping and are presented separately. Intracellular stimulation is applied using positive rectangular current pulses of $150 \mathrm{~ms}$ starting at $t=0$. Stimulus amplitudes ranged from 300 to $760 \mathrm{pA}$ which resulted in subthreshold and suprathresold responses. In the latter cases, bioelectrical activity was observed in the extracellular recording.
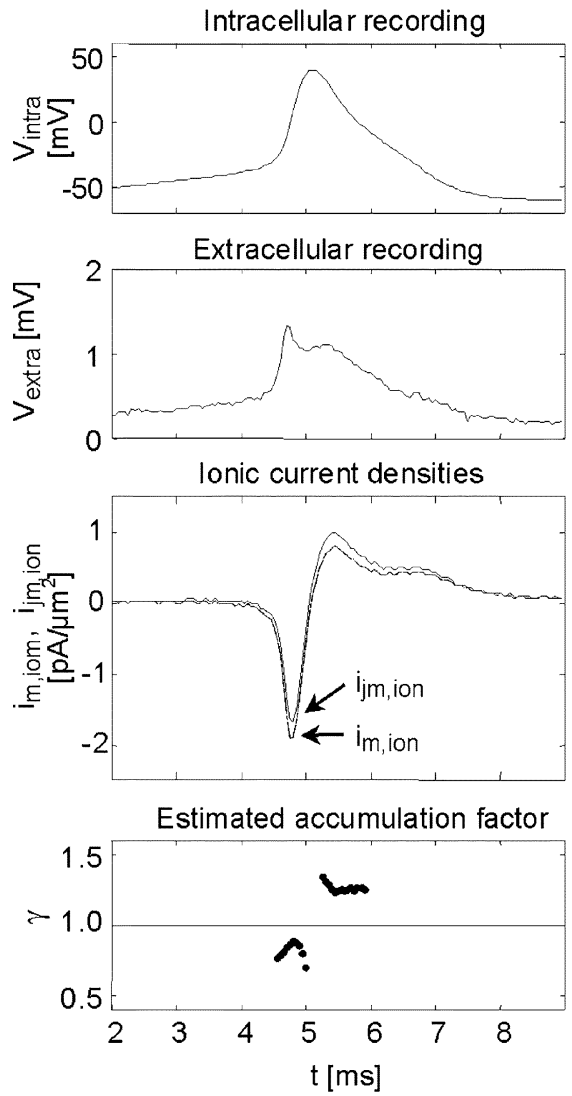

Neuron 1a

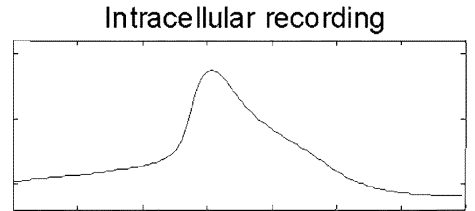

Extracellular recording

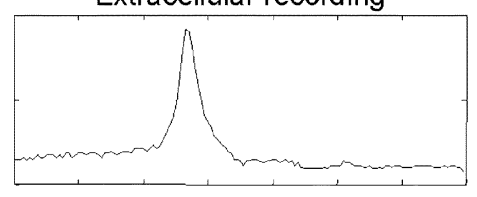

Ionic current densities

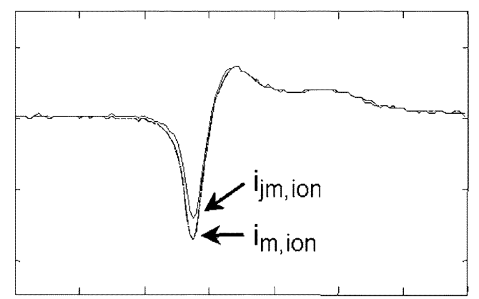

Estimated accumulation factor

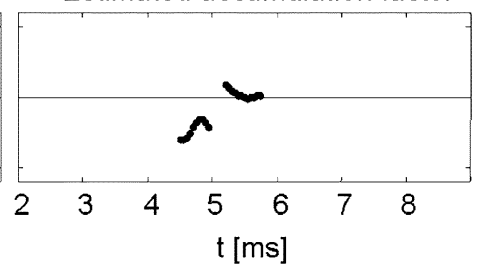

Neuron $1 \mathrm{~b}$

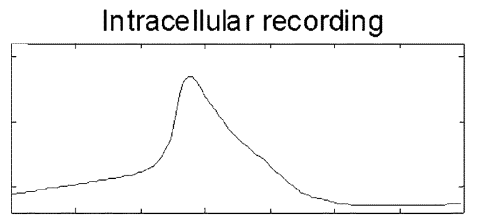

Extracellular recording

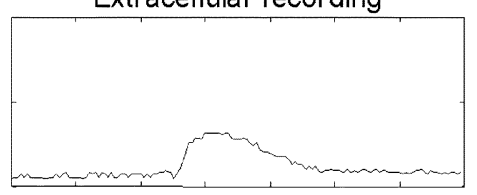

Ionic current densities

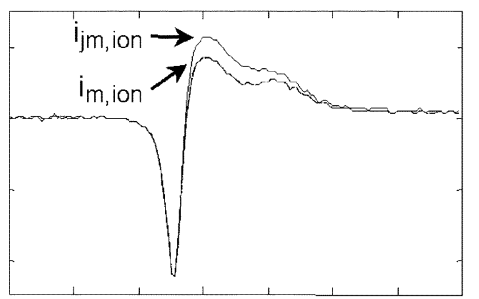

Estimated accumulation factor

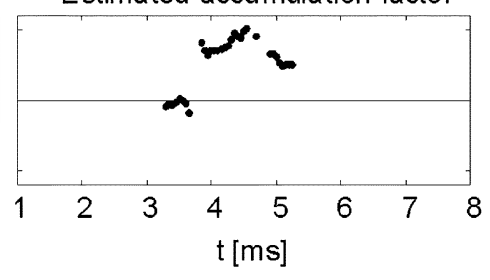

Neuron 2

Fig. 8. First row: Measured intracellular recordings due to intracellular current stimulation. Second row: Measured extracellular responses. Third row: Estimated average ionic current density, $i_{m \text {,ion }}$, and the lower membrane current density, $i_{j m, i o n}$. Fourth row: Estimated accumulation factor, $\gamma$.

separately as $1 \mathrm{a}$ and $1 \mathrm{~b}$, respectively. After initial subthreshold stimulation, intracellular action potentials are observed. All extracellular recordings consist of a passive response to the injected stimulus current. In case of suprathreshold stimulation, the extracellular recording also contains a response to the action potential in the neuron. Although the morphology of the intracellular action potentials is almost the same in both neurons, large differences in both shape and amplitude are observed between the extracellular recordings.

The measured intracellular and extracellular responses to the stimulus pulse with the highest amplitude are selected for further examination (Fig. 8). The extracellular response 
TABLE I

Parameters EXTRACTED From MEASURED InTRACELlular and EXTRACELlular ReCORDINGS (SEe Also Fig. 8)

\begin{tabular}{ccccccccc}
\hline $\begin{array}{c}\text { Neuron } \\
\#\end{array}$ & $\begin{array}{c}R_{a} \\
{[\mathrm{MÙ]}]}\end{array}$ & $\begin{array}{c}C_{m} \\
{[\mathrm{pF}]}\end{array}$ & $\begin{array}{c}A_{m} \\
{\left[\mu \mathrm{m}^{2}\right]}\end{array}$ & $\begin{array}{c}I_{\text {stim }} \\
{[\mathrm{pA}]}\end{array}$ & $\begin{array}{c}V_{e}^{(\text {passive })} \\
{[\mu \mathrm{V}]}\end{array}$ & $\begin{array}{c}\alpha \cdot R_{\text {seal }} \\
{[\mu \mathrm{V} / \mathrm{pA}]}\end{array}$ & $\begin{array}{c}R_{\text {seal }} \\
{[\mathrm{MÙ}]}\end{array}$ & $\alpha$ \\
\hline $1 \mathrm{a}$ & 11.3 & 100 & $10 \cdot 10^{3}$ & 592 & 265 & 0.45 & 4.11 & 0.11 \\
$1 \mathrm{~b}$ & 11.7 & 96 & $9.6 \cdot 10^{3}$ & 591 & 256 & 0.43 & 4.27 & 0.10 \\
2 & 6.1 & 100 & $10.1 \cdot 10^{3}$ & 760 & 128 & 0.17 & 1.73 & 0.10 \\
\hline
\end{tabular}

of neuron $1 \mathrm{~b}$ (second diagram on second row) consists of a fast peak during the rising phase of the intracellular action potential. The response of neuron 2 exhibits a slower wave of less amplitude, which occurs during the falling phase of the action potential. The response of neuron 1a looks like a linear combination of both shapes.

The access resistances, $R_{a}$, and cell capacity, $C_{m}$, are determined from the intracellular recordings. Also, the total membrane area is derived from the cell capacity and the passive response, $V_{e}^{\text {(passive) }}$, to the stimulus current was estimated just after the pulse onset (see also Fig. 7). These values are listed in Table I, together with the product of the passive contact parameters using (11). The electrical equivalent fraction of the membrane involved in the contact, $\alpha$, is computed using the sealing resistance, $R_{\text {seal }}$, which is measured using impedance spectroscopy.

The intracellular recordings were corrected for the access resistances in the patch pipette. Subsequently, the average and lower membrane ionic current densities, $\tilde{i}_{m \text {,ion }}$ and $\tilde{i}_{j m \text {,ion }}$, are computed using (12), (13), and (16). Furthermore, the estimated accumulation factor, $\gamma$, is computed using (17) for times at which $\tilde{i}_{m \text {,ion }}>0.5 \mathrm{pA} / \mu \mathrm{m}^{2}$.

For neuron $1 \mathrm{~b}, \tilde{i}_{j m \text {,ion }}$ only deviates from $\tilde{i}_{m \text {,ion }}$ during the fast peak. During this peak, $\tilde{i}_{m \text {,ion }}$ reaches a minimum of $-1.75 \mathrm{pA} / \mu \mathrm{m}^{2}$, while $\tilde{i}_{j m \text {,ion }}$ reaches a minimum of only $-1.40 \mathrm{pA} / \mu \mathrm{m}^{2}$. The estimated accumulation factor reaches a value of $\gamma=0.8$ at the minimum of the fast peak of $\tilde{i}_{m \text {,ion }}$, suggesting a $\mathrm{Na}^{+}$channel depletion in the lower membrane [cfg. Fig. 4(a)].

For neuron 2 , the deviation between $\tilde{i}_{j m \text {,ion }}$ and $\tilde{i}_{m \text {,ion }}$ occurs only during the slow wave. The maximum of $\tilde{i}_{m \text {,ion }}$ $\left(1.3 \mathrm{pA} / \mu \mathrm{m}^{2}\right)$ exceeds the maximum of $\tilde{i}_{m \text {,ion }}\left(0.81 \mathrm{pA} / \mu \mathrm{m}^{2}\right)$, which suggests an $\mathrm{K}^{+}$accumulation of $\gamma_{K}=1.4$ [cfg. Fig. 4(c)]. However, according to the simulation results this is not likely to be a true estimate. Similarly, the data from neuron 1a can be explained by simultaneous depletion of $\mathrm{Na}^{+}$and accumulation of $\mathrm{K}^{+}$. However, more accurate estimates cannot be obtained.

\section{DisCUSSION}

\section{A. Effects of Channel Distributions}

The neuron-electrode interfaces considered in this paper are very promising for neuroscientific research. For large Helisoma neurons, similarities with with electrophysiological techniques using glass pipettes (such as loose patch clamping) were already acknowledged by Regehr [2]. The simulation results and the experimental data indicate that the similarity also applies for smaller neurons, since sufficient sealing can be obtained for extracellular recording. However, a major difference with the loose patch clamp is the existence of a spatially extended sealing gap between membrane and subtrate. Furthermore, the geometry of the sealing gap is much more variable and can result in complete as well as defect sealing of the electrode. Finite-element modeling offers the advantage of easy representation of a variety of interface geometries, including complete and defect sealing. Additionally, the model presented in this paper permits a quantitative insight in the influence of channels distributions on shapes and amplitudes of extracellular recordings.

According to the simulations, the distribution of voltage gated channels plays a major role in the extracellular recording of bioelectical activity. A uniform distribution of channels results in mutual cancellation of the ionic and capacitive membrane currents (Fig. 3). Variations in the channel distribution resulted in a diverse expression of bioelectrical activity in extracellular recordings due to local cancellation errors, as demonstrated for neuron-transistor couplings by Vassanelli and Fromherz [5]. The results indicate that small variations in the channel distribution, e.g., $\gamma_{\mathrm{Na}}=0.8$, are already sufficient for extracellular recordings of hundreds of microvolts amplitude (Figs. 4 and 8).

In this paper, a uniform channel distribution in the lower membrane is assumed. Although this assumption is instructive with respect to the basic effect of accumulation and depletion of channels, it is more than likely that it does not hold for experimental situations. Based on the mobility of the channels in the membrane, as mentioned in Section I, the consequences of nonuniform channel distributions for extracellular recordings will be addressed below.

If the channels are immobilized to adhesion proteins in the membrane, the channels will be located at the focal adhesion points, which often occur at the outer edge of the sealing gap. Such channel distributions might not be very favorable for extracellular recording. Hence, when cell-substrate adhesion mechanisms might play a significant role, improvement of the neuronelectrode contact should concentrate on rejection or attraction of channels to the center of the interface by selection of suitable adhesion promotors.

When the distribution in the sealing gap is ruled by the interplay between diffusion and electrophoresis, a focus will be formed at the (electrical) center of the neuron-electrode interface.

This focus could be either a depletion or an accumulation of channels, since the direction of the current in the sealing gap depends on the initial distribution of channels. In case of an initial accumulation of $\mathrm{Na}^{+}$channels in the lower membrane, an inward current will arise during an action potential. All positively charged channels will migrate toward the center of the sealing gap, which also contributes to the accumulation of $\mathrm{Na}^{+}$channels and, in turn, to the inward current. This self-focussing effect, as proposed by Fromherz on a theoretical basis [11], would result in channel distributions which are much more favorable than the distributions caused by immobilized channels. Using FEM, the effect of any of these channel distributions on the extracellular recordings can be modeled easily. 


\section{B. Estimation of Channel Distributions}

Estimation of channel accumulation factors is performed by comparison of the lower membrane ionic current density, (14), with the average ionic current density, (15). The ratio between these current densities reflects the cancelation error in the lower membrane as a function of time. Since the lower membrane ionic current density is assumed to be uniformly distributed [(12) and (13)], the estimated accumulation factor, $\gamma$ [see (17)], explains the extracellular recording in terms of contributions from uniformly distributed channels over the lower membrane which can be depleted or accumulated.

In the simulations, the channels are also uniformly distributed over the lower membrane and accumulation factors are varied for one ion-specific channel at the time (Fig. 4). However, even for these simple distributions, estimation of channel accumulation factors appeared to be delicate due to co-occuring ion-specific membrane currents. Accumulation of $\mathrm{Na}^{+}$channels can be estimated when $\mathrm{Na}^{+}$currents dominate the ionic membrane currents during the rising phase of the action potential. Estimation of the accumulation of $\mathrm{K}^{+}$channels, however, requires additional techniques to separate the $\mathrm{K}^{+}$currents from other ionic membrane currents. The use of a voltage-clamp intracellular stimulation mode in combination with selective channel blockers might solve this problem.

Another impeding factor for true estimation of channel accumulation factors is formed by defect sealing. It was demonstrated that this geometry not only decreased the observability of bioelectrical activity due to poor passive contact parameters (Fig. 5), but also due to high-frequency leakage (Fig. 6). Further study of these effects can reveal quantitatively how this high-frequency leakage is determined by the electrode impedance, the covered fraction of the electrode area and the thickness of the sealing gap.

\section{Interpretation of Experimental Data}

The presented experimental data are anecdotal and the extracellular recordings of neuron 1 suffer from a yet unexplained change in morphology, while the intracellular action potential remained unchanged. However, no change in the quality of the patch clamp or the passive neuron-electrode contact was observed that could compromise the validity of the measurements (Table I). Furthermore, experimental data from neuron-electrode interfaces with complete sealing is sparse since these geometries are still hard to obtain. Hence, any opportunity to add more records to the literature should be taken at this stage.

Minimal attempt is made to let the modeled channel dynamics correspond to our specific experimental situation. As a result, the duration of the simulated intracellular action potential is longer than the measured action potentials. This difference is likely to be caused by the lack of fast delayed rectifier $\mathrm{K}^{+}$currents in the model, which would also explain the difference between the experimental and simulated extracellular recording due to accumulation of $\mathrm{K}^{+}$channels (Figs. 4 and 8). Except for this difference, the shapes and amplitudes of the simulated extracellular potentials show similarity with experimental recordings, which indicates that the simulation results demonstrate a general effect of channel distributions, not specific for a particular type of neuron.

Although the measurements show similarity to the simulation results and can be explained in terms of estimated accumulation factors, this is not a solid proof that the extracellular potentials are indeed an effect of channel distributions, as proposed by the model. Other mechanisms that could induce these extracellular recordings should be considered as well. As a starting point in the identification of these mechanisms, it should be recognized that any recorded extracellular potential must be due to a current density in the sealing gap (Fig. 1). If this potential is related to bioelectrical activity, the current density must flow through the lower membrane of the neuron. The simulation results, presented in this paper (Fig. 3, first column) confirm the conclusions drawn by Vassanelli and Fromherz [5], that biolectrical activity will not result in a current density in the sealing gap as long as the membrane behavior is equal over the entire neuronal surface. Hence, any extracellular recording of biolelectrical activity must result from some altered local membrane behavior, with respect to the global membrane behavior. Several causes for altered local membrane behavior can exist: 1) altered local channel densities; 2) altered local membrane capacity; 3 ) altered local ion equilibrium potentials; and 4) altered local channel activations. These causes will be addressed briefly below.

The effect of altered local channel densities is studied in this paper, and appeared a possible explanation for the extracellular recordings. Furthermore, it is a likely cause since local variations in channel densities are reported in literature and several underlying mechanisms are proposed [6]-[11].

An altered membrane capacity in the sealing gap would result in a cancelation error during the entire action potential, i.e., during both the $\mathrm{Na}^{+}$and $\mathrm{K}^{+}$current densities. Since the experimental data of neuron 2 show only cancelation errors during the falling phase of the action potential, altered membrane capacity cannot explain these extracellular recordings. Nevertheless, slight differences in the local membrane capacity can exist and their effect on the extracellular recording should be studied in more detail.

Local ion equilibrium potentials can be altered due to changes of the ion concentrations in the sealing gap, which are sometimes mentioned as a possible explanation for extracellular recordings. Since the volume of the sealing gap is very small, ion concentrations are considered to be altered easily. Since the extracellular concentration of $\mathrm{K}^{+}$is very low $(5 \mathrm{mM}$ in our experiments), the $\mathrm{K}^{+}$equilibrium potential is very sensitive for addition or removal of small amounts of $\mathrm{K}^{+}$from the sealing gap. The experimental results of neuron 2 suggest an increased outward $\mathrm{K}^{+}$current density through the lower membrane (Fig. 8). Proposing the $\mathrm{K}^{+}$equilibrium potential as an explanation for the extracellular recording, would require this potential to be more hyperpolarising and, hence, require a decreased $\mathrm{K}^{+}$concentration in the sealing gap. Similarly, a decreased concentration of $\mathrm{Na}^{+}$in the sealing gap would be required for explaining the decreased inward $\mathrm{Na}^{+}$currents suggested by the experimental data from neuron 1 . These required permanently or temporarily altered ion concentrations in the sealing gap are not necessarily consistent with the mechanisms for addition or removal of ions, such as the inward $\mathrm{Na}^{+}$and 
outward $\mathrm{K}^{+}$currents during an action potential, the NaK-pump and diffusion and electrophoresis from outside the sealing gap. Further research should be conducted on this topic.

Altered local activation of voltage-sensitive channels, is primairily caused by local membrane potentials. Due to a high resisitivity of the sealing gap, current densities through the lower membrane can result in substantially altered local membrane potentials and, hence, channel activations. Since the model, presented in this paper, computes local membrane potentials and channel activations, this mechanism is fully represented. However, the simulations with uniform channel distributions demonstrate that the local membrane potential in the sealing gap does not alter the behavior of the lower membrane sufficiently for recording bioelectrical activity.

As a preliminary conclusion, it can be stated that the channel distributions are the most simple and the most likely explanation for the extracellular recordings. However, further research should be conducted to obtain a final conclusion on the effects of other mechanisms. Of course, an independent method for determination of channel distributions in the lower membrane, such as fluorescent labeling, would provide strong evidence about their role in extracellular recording.

\section{ACKNOWLEDGMENT}

The authors would like to thank K. Peter and M. Deenen for their excellent support of the culturing activities in their lab and M. Goedbloed for the fabrication of the MEAs used in the experiments.

\section{REFERENCES}

[1] G. W. Gross, "Simultaneous single unit recording in vitro with a photoetched laser deinsulated gold multimicroelectrode surface," IEEE Trans. Biomed. Eng., vol. BME-26, pp. 273-279, 1979.

[2] W. G. Regehr, J. Pine, C. S. Cohan, M. D. Mischke, and D. W. Tank, "Sealing cultured invertebrate neurons to embedded dish electrodes facilitates long-term stimulation and recording," J. Neurosci. Meth., vol. 30, pp. 91-106, 1989.

[3] M. Bove, M. Grattarola, S. Martinoia, and G. Verreschi, "Interfacing cultured neurons to planar substrate microelectrodes: Characterization of the neuron-to-microelectrode junction," Bioelectrochem. Bioenerg., vol. 38, pp. 255-265, 1995.

[4] L. J. Breckenridge, R. J. Wilson, P. Connolly, A. S. Curtis, J. A. Dow, S. E. Blackshaw, and C. D. Wilkinson, "Advantages of using microfabricated extracellular electrodes for in vitro neuronal recording," $\mathrm{J}$. $\mathrm{Neu}$ rosci. Res., vol. 42, pp. 266-76, 1995.

[5] S. Vassanelli and P. Fromhertz, "Tranistor records of excitable neurons from rat brain," Appl. Phys. A, vol. 66, pp. 549-463, 1998.

[6] B. Hille, Ionic Channels of Excitable Membranes, 2nd ed. Sunderland, MA: Sinauer Assoc., Inc., 1992.

[7] M. J. Titmus and D. S. Faber, "Altered excitability of goldfish mauthner cell following axotomy. II localization and ionic basis," J. Neurphysiol., vol. 55, pp. 1440-1454, 1986.

[8] K. J. Angelides, L. W. Elmer, D. Loftus, and E. Elson, "Distribution and lateral mobility of voltage-dependent sodium channels in neurons [published erratum appears in J. Cell Biol. 1989 May;108(5):preceding 2001]," J. Cell Biol., vol. 106, pp. 1911-1925, 1988.

[9] M. Siegel, E. Marder, and L. F. Abbott, "Activity-dependent current distributions in model neurons," in Proc. Nat. Acad. Sci. USA, vol. 91, 1994, pp. $11308-11312$.
[10] S. S.-H. Wang and S. Thompson, "A-type potassium channel clusters revealed using new statistical analysis of loose patch data," Biophys. J., vol. 63, pp. 1018-1025, 1992.

[11] P. Fromherz, "Self-focusing of ion channels in cell adhesion," Phys. Rev. Lett. E, vol. 52, pp. 1303-1305, 1995.

[12] J. R. Buitenweg, W. L. C. Rutten, and E. Marani, "Geometry-based finite element modeling of the electrical contact between a cultured neuron and a microelectrode," IEEE Trans. Biomed. Eng., vol. 50, 2003.

[13] A. Marty and E. Neher, "Tight seal whole cell recording," in Single Channel Recording, B. Sakmann and E. Neher, Eds. New York: Plenum, 1983, pp. 107-122.

[14] M. D. Baker and H. Bostock, "Low-threshold, persistent sodium current in rat large dorsal root ganglion neurons in culture," J. Neurophysiol., vol. 77, pp. 1503-1513, 1997.

[15] S. A. Fedulova, D. V. Vasilyev, and N. S. Veselovsky, "Voltage-operated potassium currents in the somatic membrane of rat dorsal root ganglion neurons: Ontogenetic aspects," Neurosci., vol. 85, pp. 497-508, 1998.

[16] Y. S. Kim, Y. K. Shin, C. S. Lee, and J.-H. Song, "Block of sodium currents in rat dorsal roots ganglion neurons by diphenhydramine," Brain Res., vol. 881, pp. 190-198, 2000.

[17] B. Everill, M. A. Rizzo, and J. D. Kocsis, "Morphologically identified cutaneous afferent DRG neurons express three different potassium currents in varying proportions," J. Neurophysiol., vol. 79, pp. 1814-1824, 1998

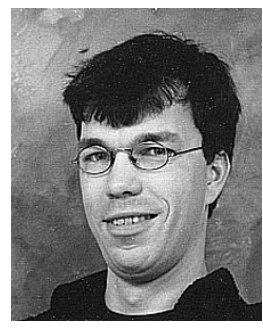

Jan Reinoud Buitenweg received the M.Sc. degree in biomedical engineering from the University of Twente, Enschede, The Netherlands, in 1998. He received the $\mathrm{Ph} . \mathrm{D}$. degree from the same institution in 2001 after studying the electrical behavior of the interface between cultured neurons and substrate embedded microelectrodes.

At present, he is an Assistant Professor with the University of Twente, with research interests in information exchange with the neuromuscular system.

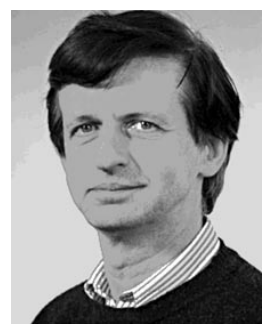

Wim L. C. Rutten received the Ph.D. degree in experimental physics from Leiden University, Leiden, The Netherlands, in 1979. Thereafter, he studied the auditory system at the ENT Department of Leiden University Hospital.

Since 1985, he has been with the BMTI/Faculty of Electrical Engineering of the University of Twente, Enschede, The Netherlands, as Assistant and Associate Professor of Biomedical Control Systems. His present research interests are neurotechnology (neuprocessing, and bioelectricity. roelectronic interfaces, and cultured probes), signal

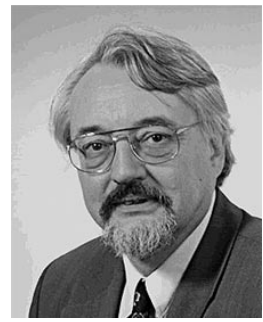

Enrico Marani received the $\mathrm{Ph} . \mathrm{D}$. degree in neuroanatomy from Leiden University, Leiden, The Netherlands, in 1982

Thereafter, he became Head of the Neuroregulation Group, Department of Physiology, Leiden University. Since 1997, he is part-time Professor in neurophysiology at the BMTI/Faculty of Electrical Engineering of the University of Twente, Enschede, The Netherlands. His present research interests are neuroregulation and neurotechnology, especially in the field of nerve regeneration. 